\title{
Characterization of Catalyst Materials for Production of Aerospace Fuels
}

Due to environmental, economic, and security issues, there is a greater need for cleaner alternative fuels. There will undoubtedly be a shift from crude oil to non-petroleum sources as a feedstock for aviation (and other transportation) fuels. Additionally, efforts are concentrated on reducing costs coupled with fuel production from non-conventional sources. One solution to this issue is Fischer-Tropsch gas-to-liquid technology. Fischer-Tropsch processing of synthesis gas $\left(\mathrm{CO} / \mathrm{H}_{2}\right)$ produces a complex product stream of paraffins, olefins, and oxygenated compounds such as alcohols and aldehydes. The Fisher-Tropsch process can produce a cleaner diesel oil fraction with a high cetane number (typically above 70) without any sulfur or aromatic compounds. This process is most commonly catalyzed by heterogeneous (in this case, silver and platinum) catalysts composed of cobalt supported on alumina or unsupported alloyed iron powders. Physisorption, chemisorptions, scanning electron microscopy (SEM), and energy dispersive spectroscopy (EDS) are described to better understand the potential performance of Fischer-Tropsch cobalt on alumina catalysts promoted with silver and platinum. The overall goal is to preferentially produce C8 to C18 paraffin compounds for use as aerospace fuels. Progress towards this goal will eventually be updated and achieved by a more thorough understanding of the characterization of catalyst materials. This work was supported by NASA's Subsonic Fixed Wing and In-situ Resource Utilization projects. 


\section{Characterization of Catalyst Materials for Production of Aerospace Fuels}

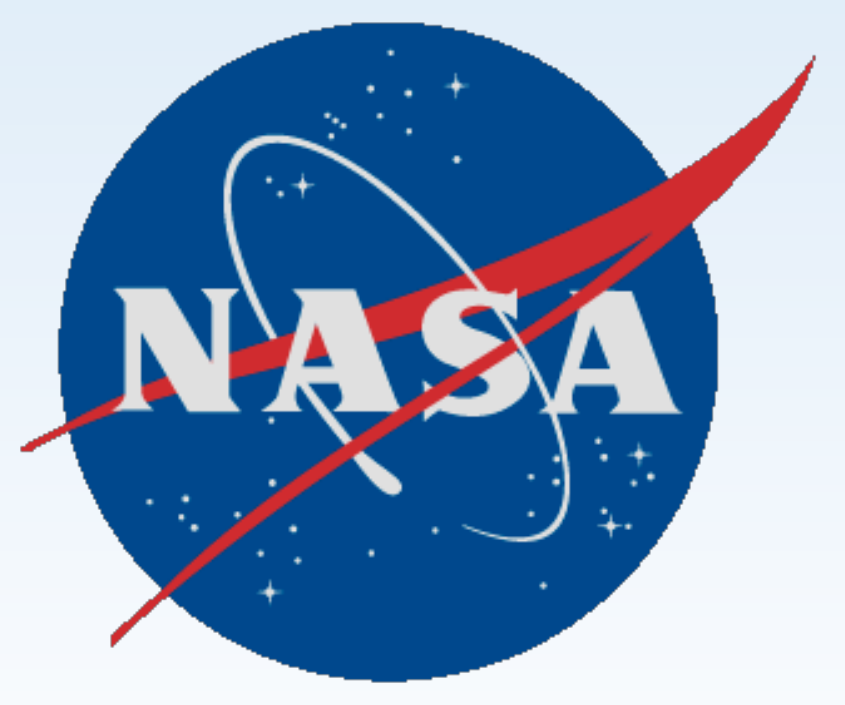

$9^{\text {th }}$ IECEC San Diego, CA August 2, 2011

\author{
Lauren M. Best \\ NASA Glenn Research Center \\ and \\ Ana B. De La Ree \\ UNCFSP/NASA GRC \\ Aloysius F. Hepp \\ NASA Glenn Research Center
}




\section{Overview}

\section{Introduction}

- Motivation

- Fischer-Tropsch Background

\section{Alternative Fuels Laboratory}

\section{Catalyst Synthesis Methodology}

\section{Characterization}

- Brunauer, Emmett, Teller (BET) Surface Area

- Temperature-Programmed Reduction (TPR)

- Scanning Electron Microscopy (SEM)/Energy Dispersive Spectroscopy (EDS)

\section{Results \& Conclusion}




\section{Motivation}
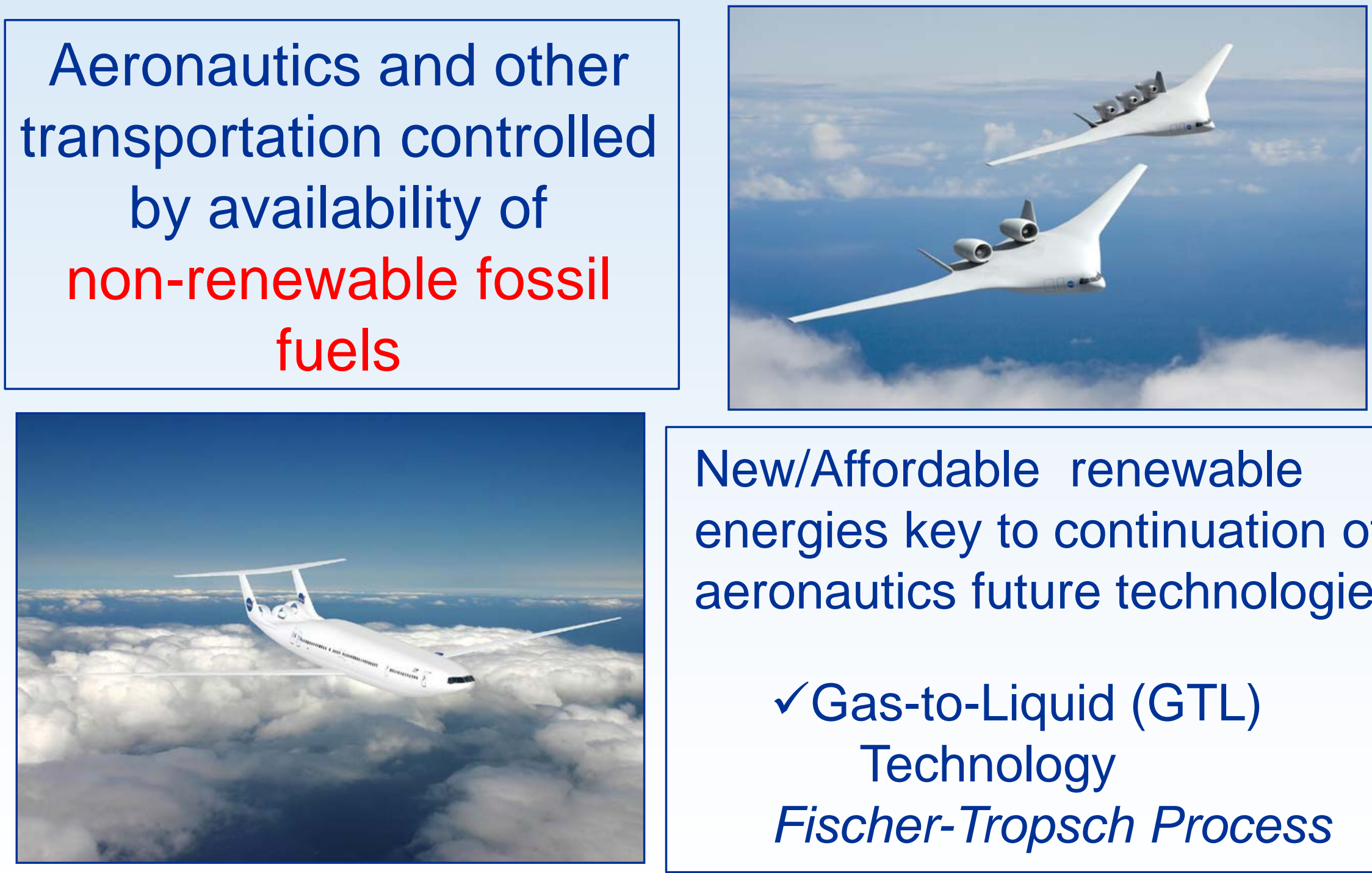

New/Affordable renewable energies key to continuation of aeronautics future technologies

\section{$\checkmark$ Gas-to-Liquid (GTL) Technology \\ Fischer-Tropsch Process}




\section{Why Gas-to-Liquid Technology (GTL)?}

\section{Fischer-Tropsch Synthesis}

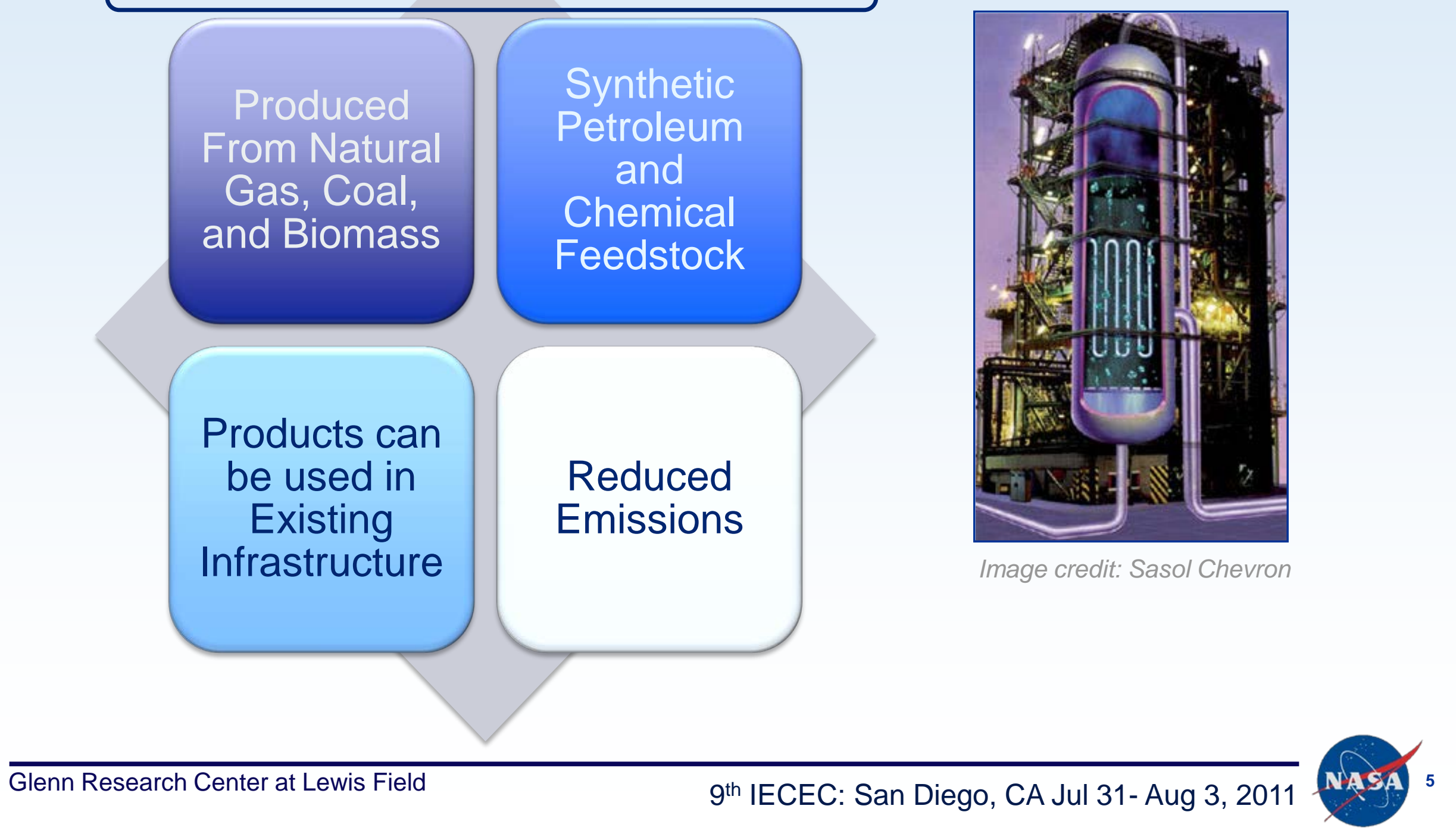




\section{Fischer-Tropsch Synthesis}

$$
\begin{gathered}
(2 \mathrm{n}+1) \mathrm{H}_{2}+\mathrm{nCO} \stackrel{\text { Catalyst }}{\longrightarrow} \mathrm{C}_{\mathrm{n}} \mathrm{H}_{(2 \mathrm{n}+2)}+{ }_{\mathrm{n}} \mathrm{H}_{2} \mathrm{O} \\
\Delta \mathrm{H}=-49 \mathrm{kcal} / \mathrm{mol}(\mathrm{n}=1)
\end{gathered}
$$

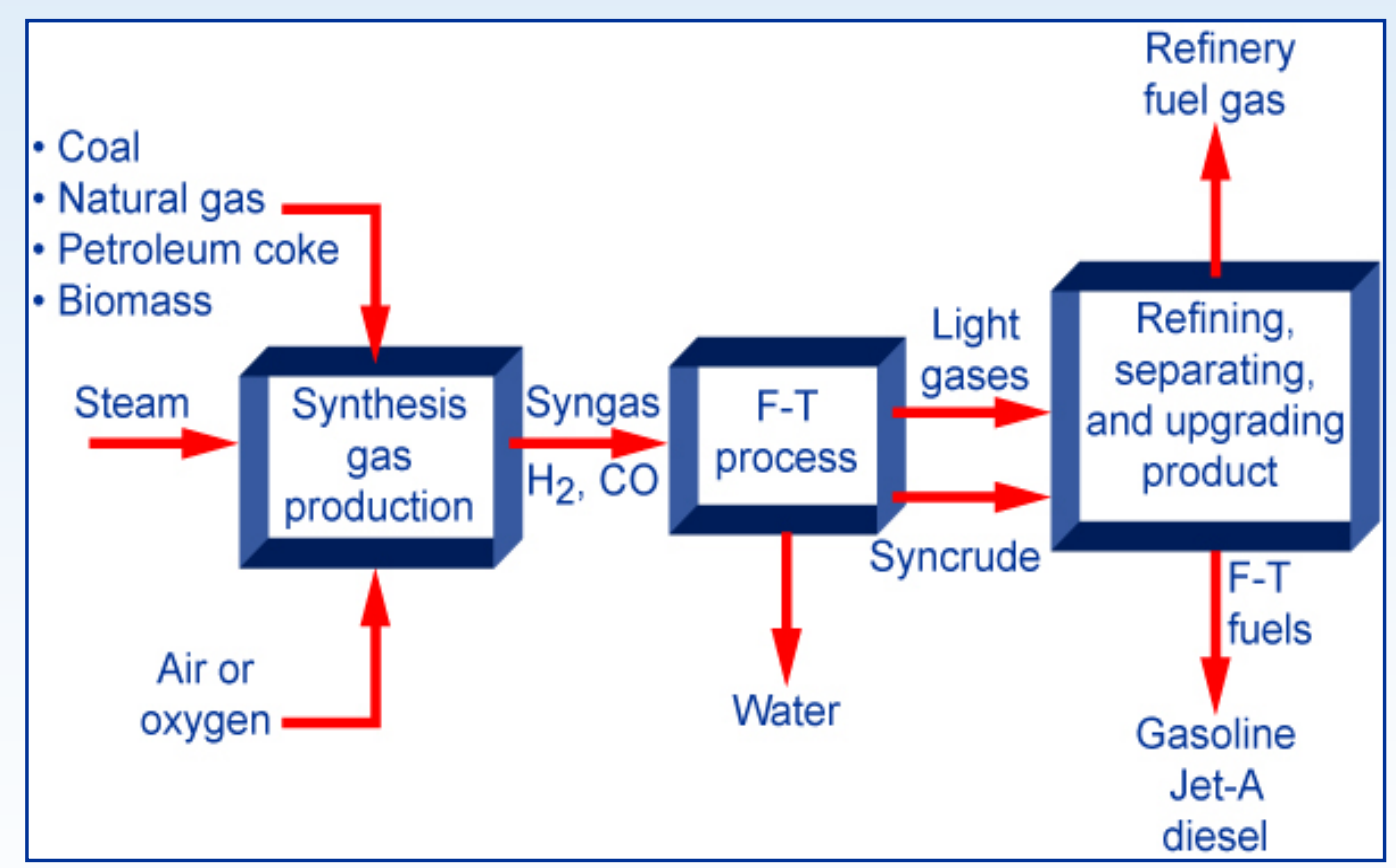

FTS upgrades syn-gas to a wide array of products:

- Hydrocarbons

- Oxygenated compounds

- Alcohols

Significant Alternative Fuel Source - Products can be converted to useful aviation fuel ( $\mathrm{C}_{8}-\mathrm{C}_{18}$ hydrocarbon chains) 


\section{Fischer-Tropsch Catalysts}

Metallic catalyst is needed to facilitate reaction between $\mathrm{CO}$ and $\mathrm{H}_{2}$

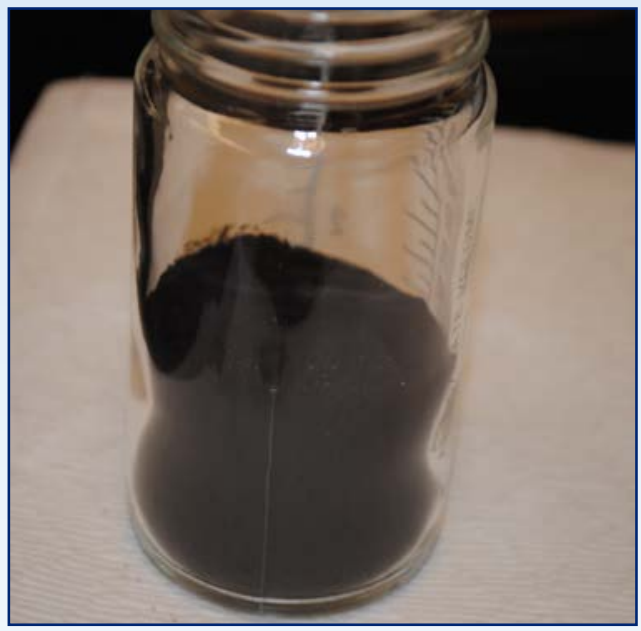

NASA cobalt catalyst $\checkmark$ Most active metals: $\mathrm{Co}, \mathrm{Fe}, \mathrm{Ru}, \mathrm{Ni}$

$\checkmark$ Catalyst is vital to performance of FT reaction

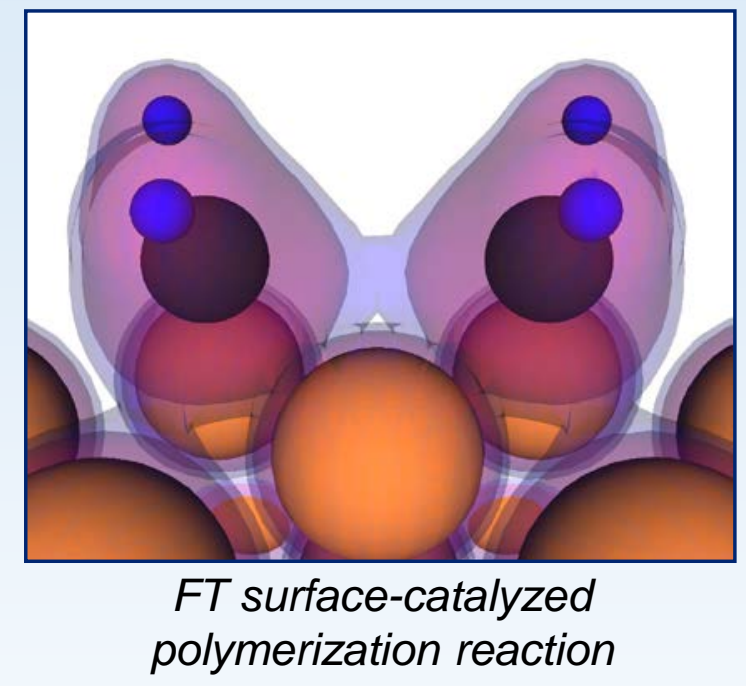

Image credit: Univ. of Wisconsin

Commercial Applications - FT synthesis utilizes Co and Fe due to lower costs

$\checkmark$ Co highly active - used for high $\mathrm{H}_{2}$ : $\mathrm{CO}$ ratio (natural gas) $\checkmark$ Fe used for low quality feedstocks (due to water-gas-shift activity)

\section{NASA GRC Catalysis Team - Cobalt Catalyst Research}




\section{Cobalt FT Catalysts and Promoters}

Cobalt catalysts supported on high surface area binders

(such as alumnia $-\mathrm{Al}_{2} \mathrm{O}_{3}$ or silica $-\mathrm{SiO}_{2}$ )

$\checkmark$ NASA GRC Research - Cobalt/Alumnia Catalysts

\section{$\checkmark$ Promoters -}

Transition Metals $\checkmark$ Promoters Benefits: oEnhance catalytic properties o Increase cobalt oxide reducibility o Stabilize catalyst

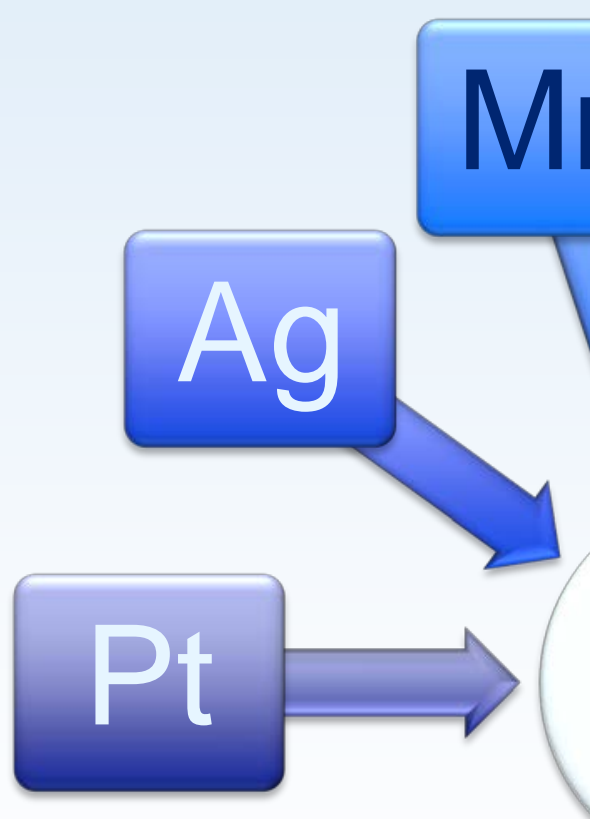

Mn and Ag considered due to low cost vs. Pt-group metals! 


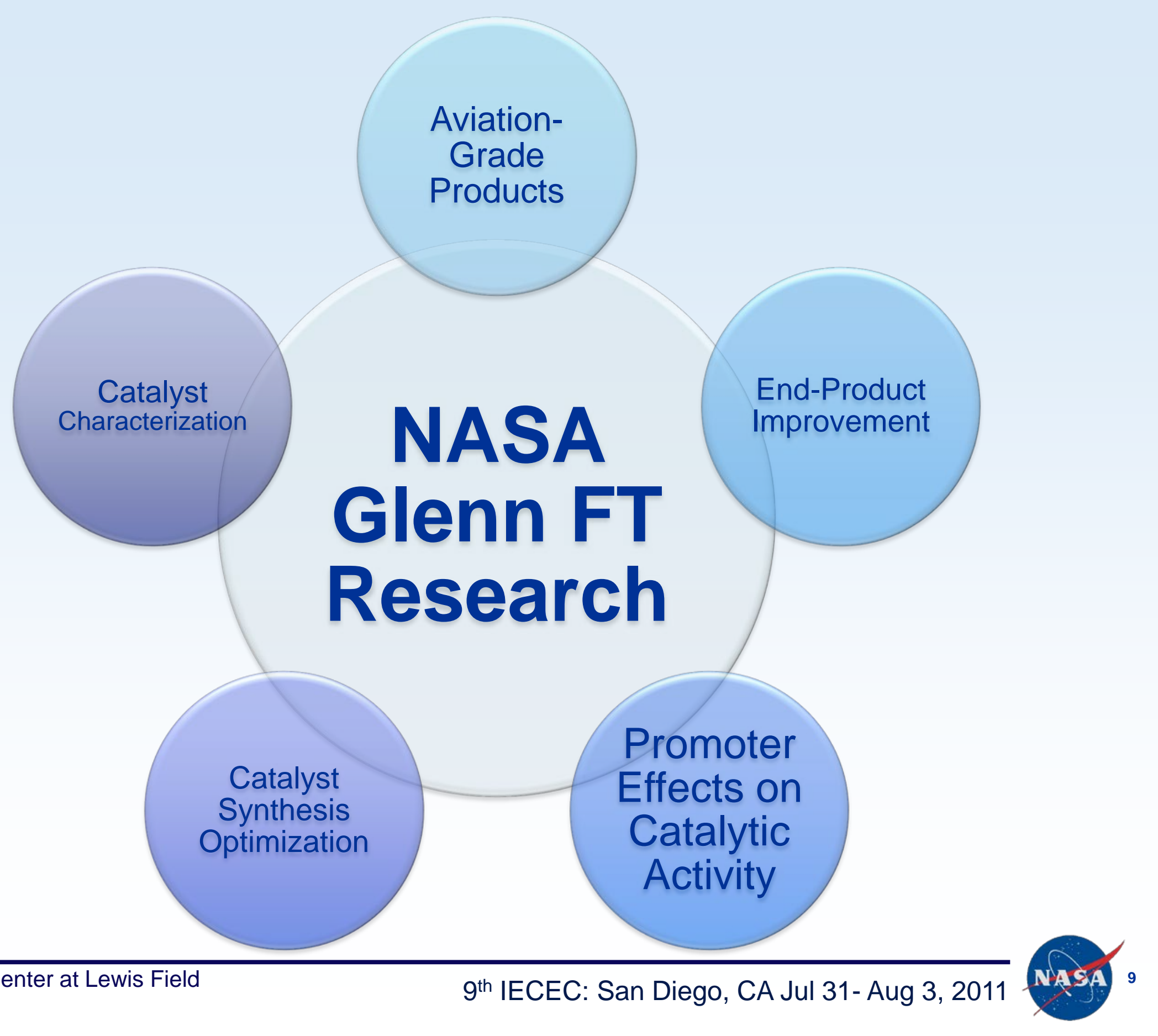




\section{NASA GRC Alternative Fuels Laboratory}

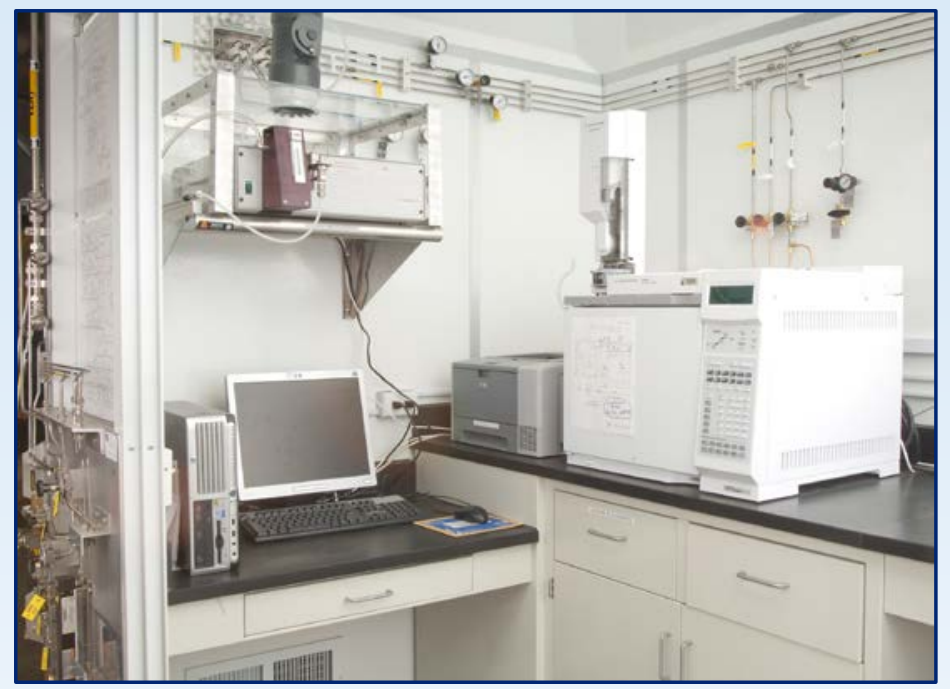

GC Work Area

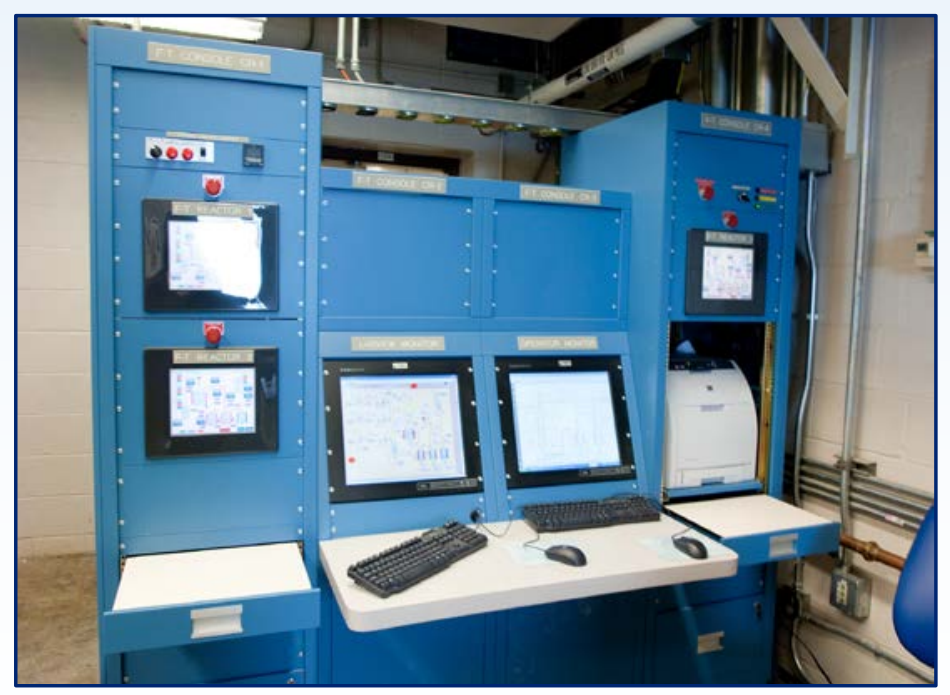

- \$3 Million facility, opened in 2010

- 3 CSTR FT Reactors

- Automated product analysis capabilities (GC)

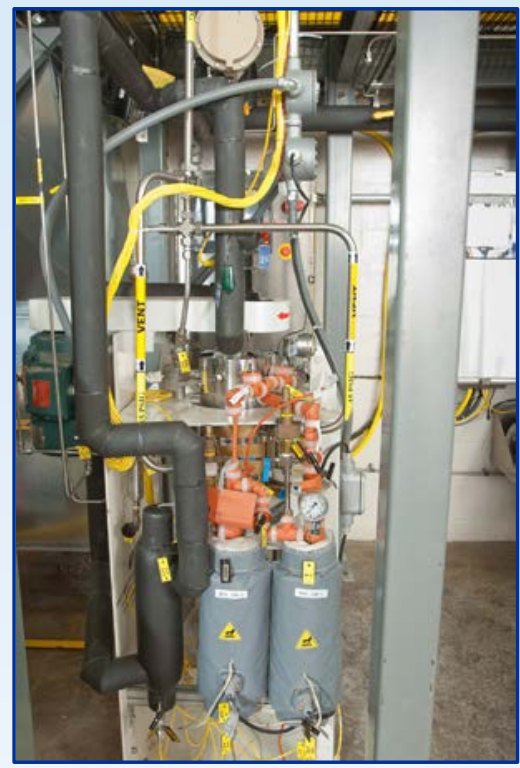

Control Room

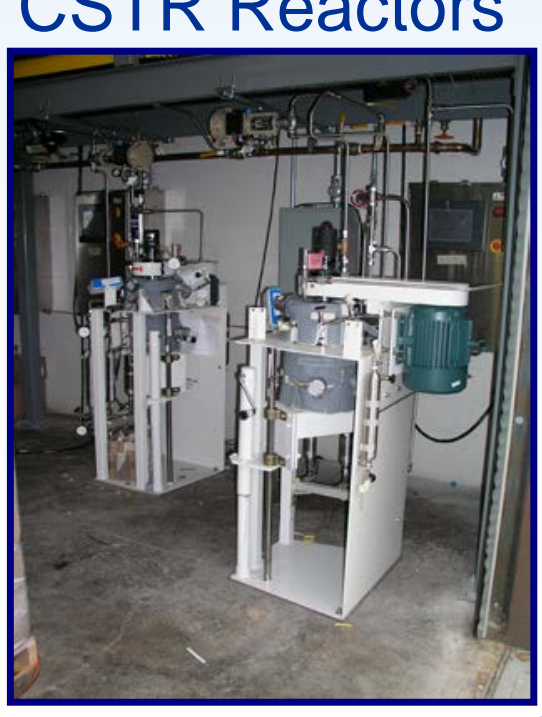




\section{Catalyst Synthesis}

Cobalt Nitrate $\left(\mathrm{Co}\left(\mathrm{NO}_{3}\right)_{2} \cdot 6 \mathrm{H}_{2} \mathrm{O}\right)$ solution added dropwise to Alumnia $\left(\mathrm{Al}_{2} \mathrm{O}_{3}\right)$
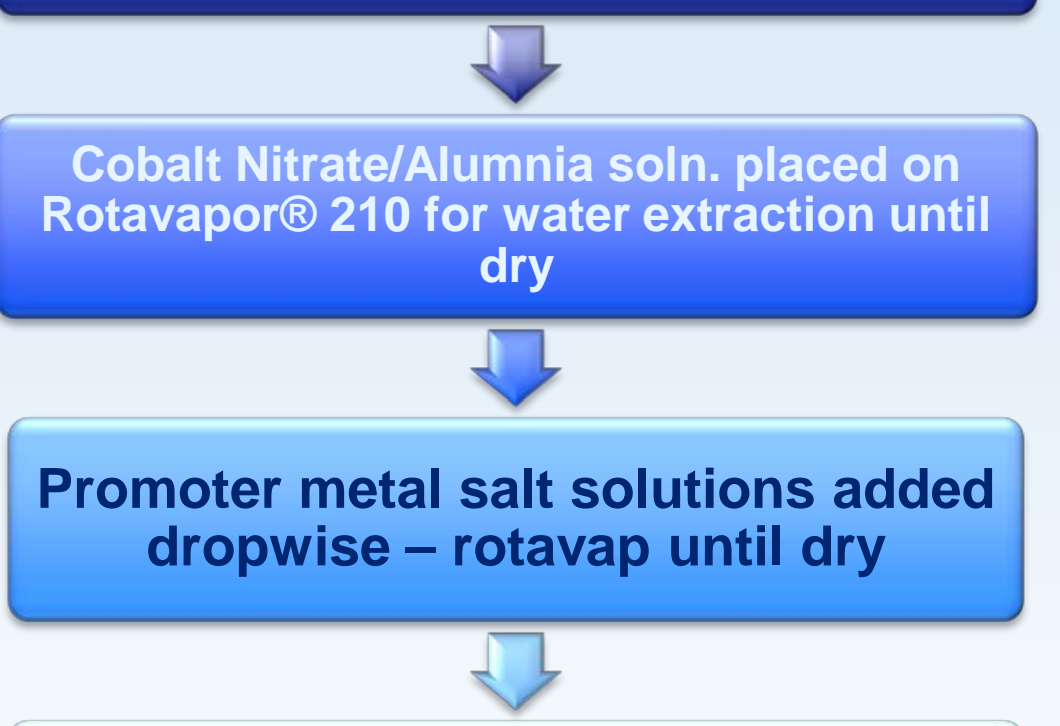

Catalyst calcinated - left with Co/Promoter/Al ${ }_{2} \mathrm{O}_{3}$

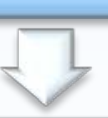

Characterization

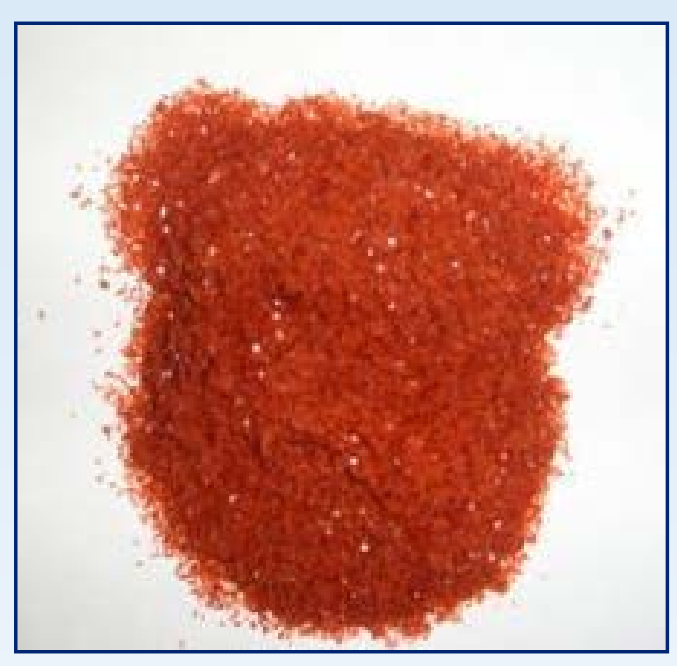

Cobalt Nitrate

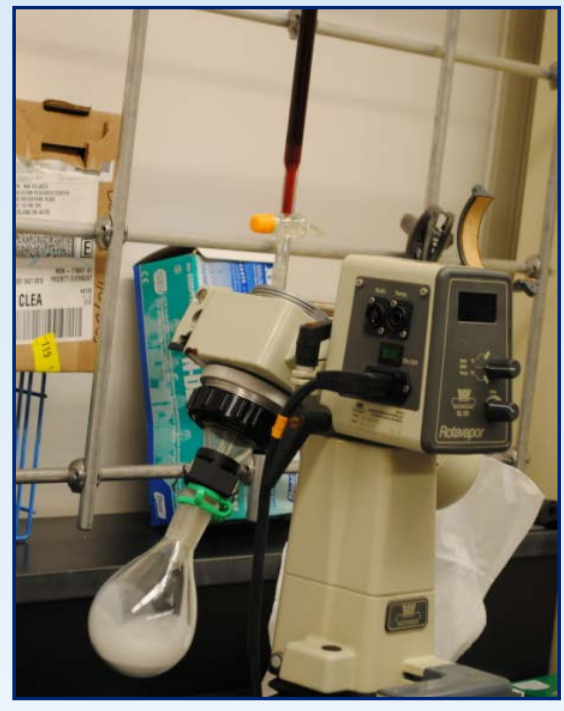

$\left(\mathrm{Co}\left(\mathrm{NO}_{3}\right)_{2} \cdot 6 \mathrm{H}_{2} \mathrm{O}\right)$ soln. added dropwise

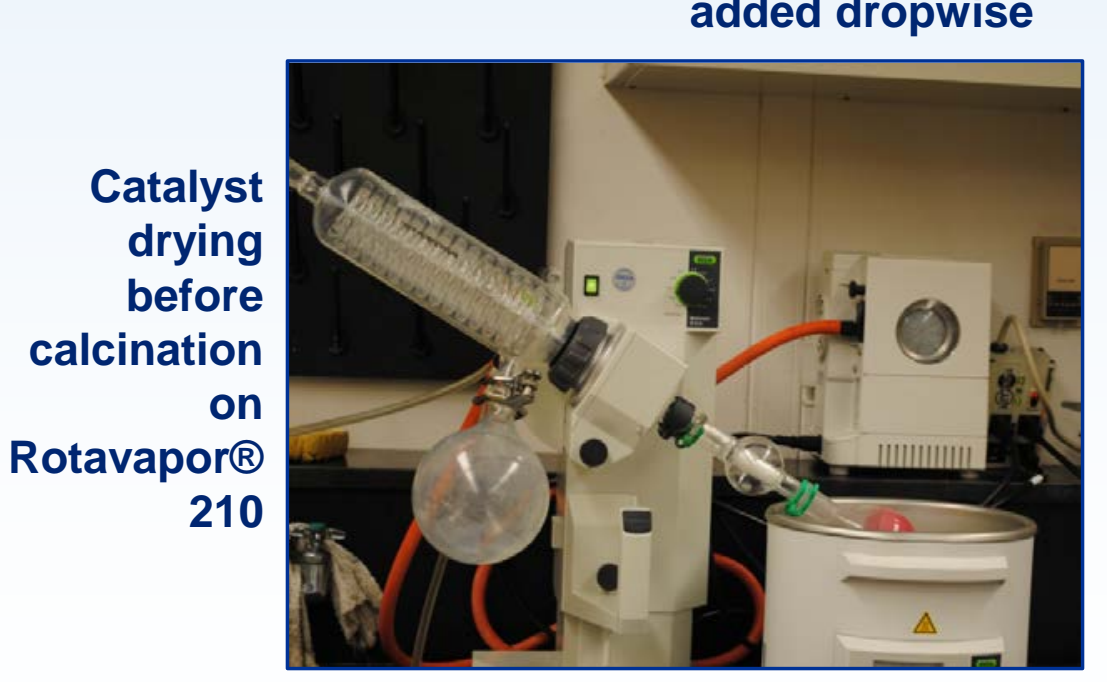

Glenn Research Center at Lewis Field

$9^{\text {th }}$ IECEC: San Diego, CA Jul 31- Aug 3, 2011 


\section{Catalyst Characterization}

Characterization Goal: Understand surface of catalyst at reaction specific conditions

$\checkmark$ Heterogeneous catalyst that can generate specific range of hydrocarbons needed

- Brunauer, Emmett, and Teller surface area analysis (BET) - surface adsorption and catalytic activity/unit area

- Temperature-Programmed Reduction (TPR) - catalyst behavior based on material composition

- Scanning Electron Microscopy (SEM)/Energy Dispersive Spectroscopy (EDS) - material composition and surface properties 


\section{Characterization Instruments}

Temperature-Programmed Reduction (TPR)

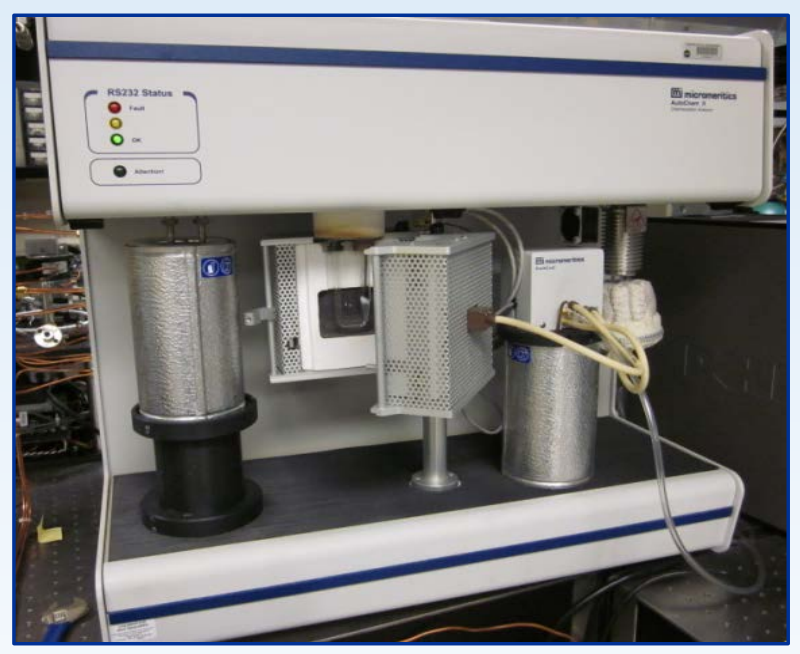

Micromeritics AutoChem II 2920
Brunauer, Emmett, and Teller Surface Area Analysis (BET)

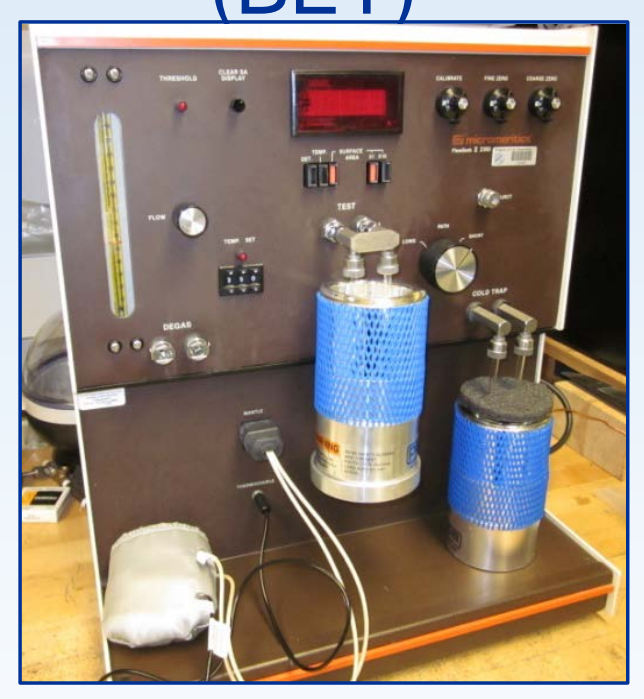

Micromeritics FlowSorb II 2300

Scanning Electron Microscopy(SEM)/Energy Dispersive Spectroscopy (EDS) - Hitachi S-3000N 


\section{Catalyst Study}

\section{$\checkmark$ In this study, unpromoted and promoted (Pt and Ag)}

\section{catalysts will be compared}

\section{$\checkmark P t$ and Ag will be also be compared to evaluate economical promoter options}

Table 1. Samples of Promoted/Unpromoted Co/Alumina Catalysts Prepared at NASA GRC

\begin{tabular}{|c|c|c|c|c|c|}
\hline$\underset{\#}{\text { Sample }}$ & Promoter & $\begin{array}{l}\text { ICP-AES Element Analysis } \\
\text { (Galbraith Laboratories, Inc.) }\end{array}$ & $\begin{array}{l}\text { Energy Dispersive } \\
\text { Spectroscopy (EDS) }\end{array}$ & $\begin{array}{c}\text { Surface } \\
\text { Area } \\
\left(\mathrm{m}^{2} / \mathrm{g}\right)\end{array}$ & $\begin{array}{c}\text { Reduction } \\
\text { Temperature }\left({ }^{\circ} \mathrm{C}\right)\end{array}$ \\
\hline 1 & None & $21.6 \% \mathrm{Co} / \mathrm{Al}_{2} \mathrm{O}_{3}$ & $30.3 \% \mathrm{Co} / \mathrm{Al}_{2} \mathrm{O}_{3}$ & 126.3 & 350 \\
\hline 2 & None & $9.31 \% \mathrm{Co} / \mathrm{Al}_{2} \mathrm{O}_{3}$ & $9.45 \% \mathrm{Co} / \mathrm{Al}_{2} \mathrm{O}_{3}$ & 142.4 & 335 \\
\hline 3 & None & $31.7 \% \mathrm{Co} / \mathrm{Al}_{2} \mathrm{O}_{3}$ & $47.2 \% \mathrm{Co} / \mathrm{Al}_{2} \mathrm{O}_{3}$ & 108.7 & 436 \\
\hline 4 & $\mathrm{Pt}$ & $21.5 \% \mathrm{Co} / 0.845 \% \mathrm{Pt}^{-} \mathrm{Al}_{2} \mathrm{O}_{3}$ & $25.4 \% \mathrm{Co} / 2.57 \% \mathrm{Pt} / \mathrm{Al}_{2} \mathrm{O}_{3}$ & 123.7 & 254 \\
\hline 5 & $\mathrm{Pt}$ & $20.9 \% \mathrm{Co} / 0.397 \% \mathrm{Pt}^{2} \mathrm{Al}_{2} \mathrm{O}_{3}$ & $24.1 \% \mathrm{Co} / 1.49 \% \mathrm{Pt} / \mathrm{Al}_{2} \mathrm{O}_{3}$ & 106.6 & 349 \\
\hline 6 & $\mathrm{Pt}$ & $24.8 \% \mathrm{Co} / 0.459 \% \mathrm{Pt} / \mathrm{Al}_{2} \mathrm{O}_{3}$ & $34.8 \% \mathrm{Co} / 2.30 \% \mathrm{Pt}^{-} / \mathrm{Al}_{2} \mathrm{O}_{3}$ & 115.9 & 265 \\
\hline 7 & $\mathrm{Ag}$ & $21.0 \% \mathrm{Co} / 0.806 \% \mathrm{Ag} / \mathrm{Al}_{2} \mathrm{O}_{3}$ & $25.9 \% \mathrm{Co} / 1.31 \% \mathrm{Ag} / \mathrm{Al}_{2} \mathrm{O}_{3}$ & 118.2 & 275 \\
\hline 8 & $\mathrm{Ag}$ & $23.6 \% \mathrm{Co} / 0.278 \% \mathrm{Ag} / \mathrm{Al}_{2} \mathrm{O}_{3}$ & $33.3 \% \mathrm{Co} / 2.19 \% \mathrm{Ag} / \mathrm{Al}_{2} \mathrm{O}_{3}$ & 109.4 & 369 \\
\hline 9 & $\mathrm{Ag}$ & $22.9 \% \mathrm{Co} / 0.510 \% \mathrm{Ag} / \mathrm{Al}_{2} \mathrm{O}_{3}$ & $26.7 \% \mathrm{Co} / 1.63 \% \mathrm{Ag} / \mathrm{Al}_{2} \mathrm{O}_{3}$ & 117.6 & 337 \\
\hline
\end{tabular}




\section{Promoted vs. Unpromoted Catalysts}
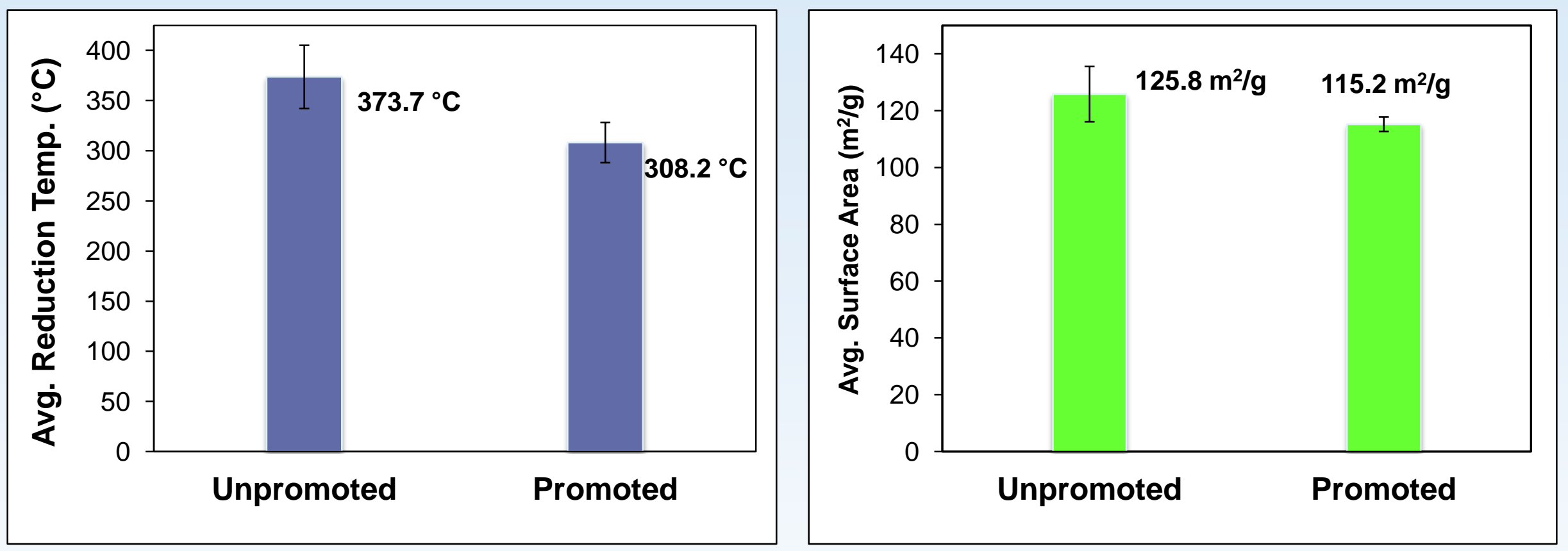

$\checkmark$ Addition of promoter decreases the necessary activation temperature of catalyst

$\checkmark$ Since cobalt fills porous space of catalyst, promoter does not reduce surface area by significant amount

*For higher accuracy purposes, ICP-AES data was used 


\section{Cobalt Loading and Catalyst Surface Area}

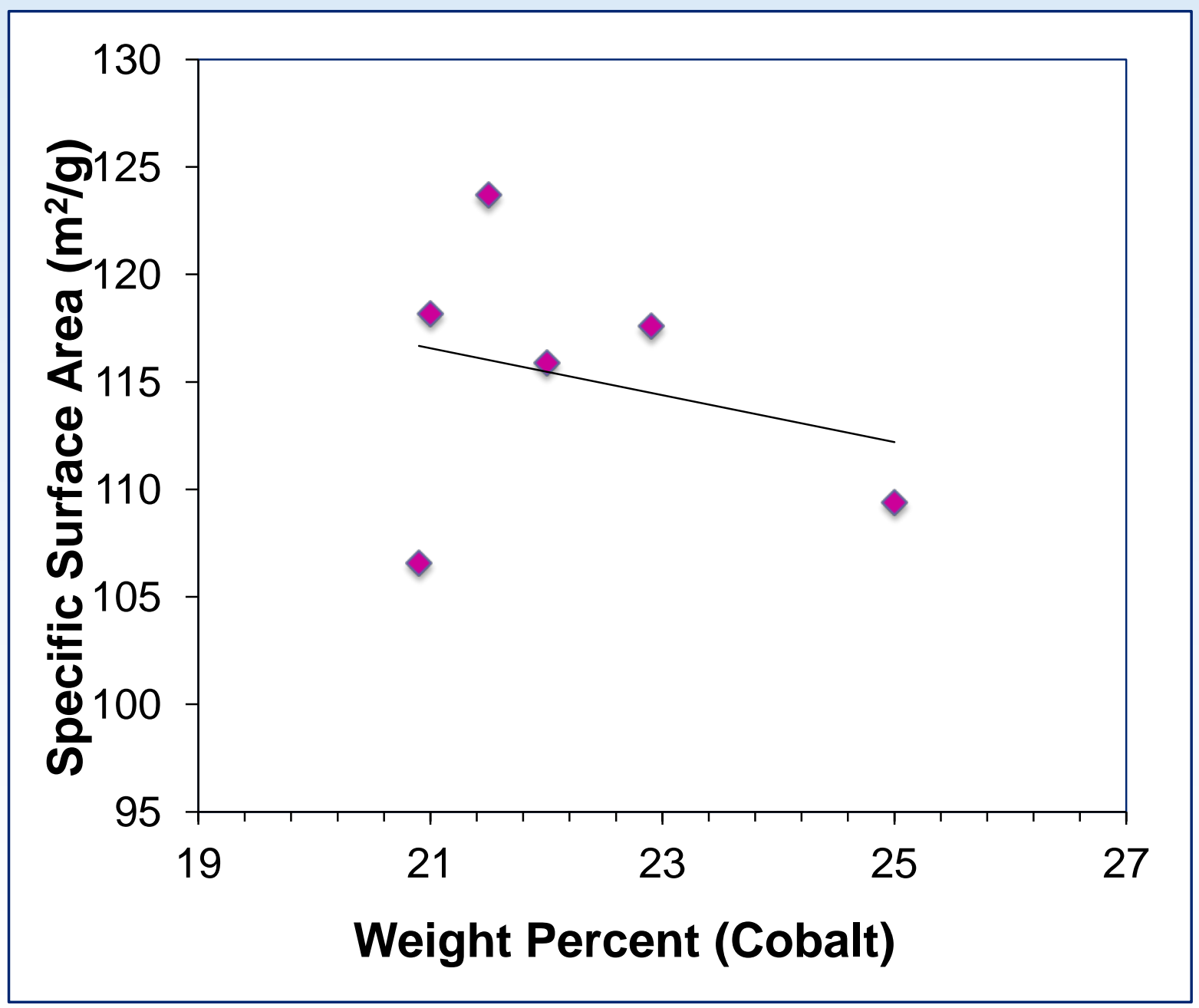

$\checkmark$ Weight percent of cobalt loading analyzed

$\checkmark$ Downward trend with regards to $\mathrm{Co} \%$ and surface area

$\checkmark$ Additional Co fills porous space of $\mathrm{Al}_{2} \mathrm{O}_{3}$ 


\section{Platinum \& Silver and Reduction Temperature}

$\checkmark$ Addition of promoters reduces reduction temperature

$\checkmark$ As wt.\% of promoter $\uparrow$ temperature $\downarrow$

$\checkmark$ Platinum has greater effect on $T$ than silver

$\checkmark$ Temp. reduction still significant with Ag

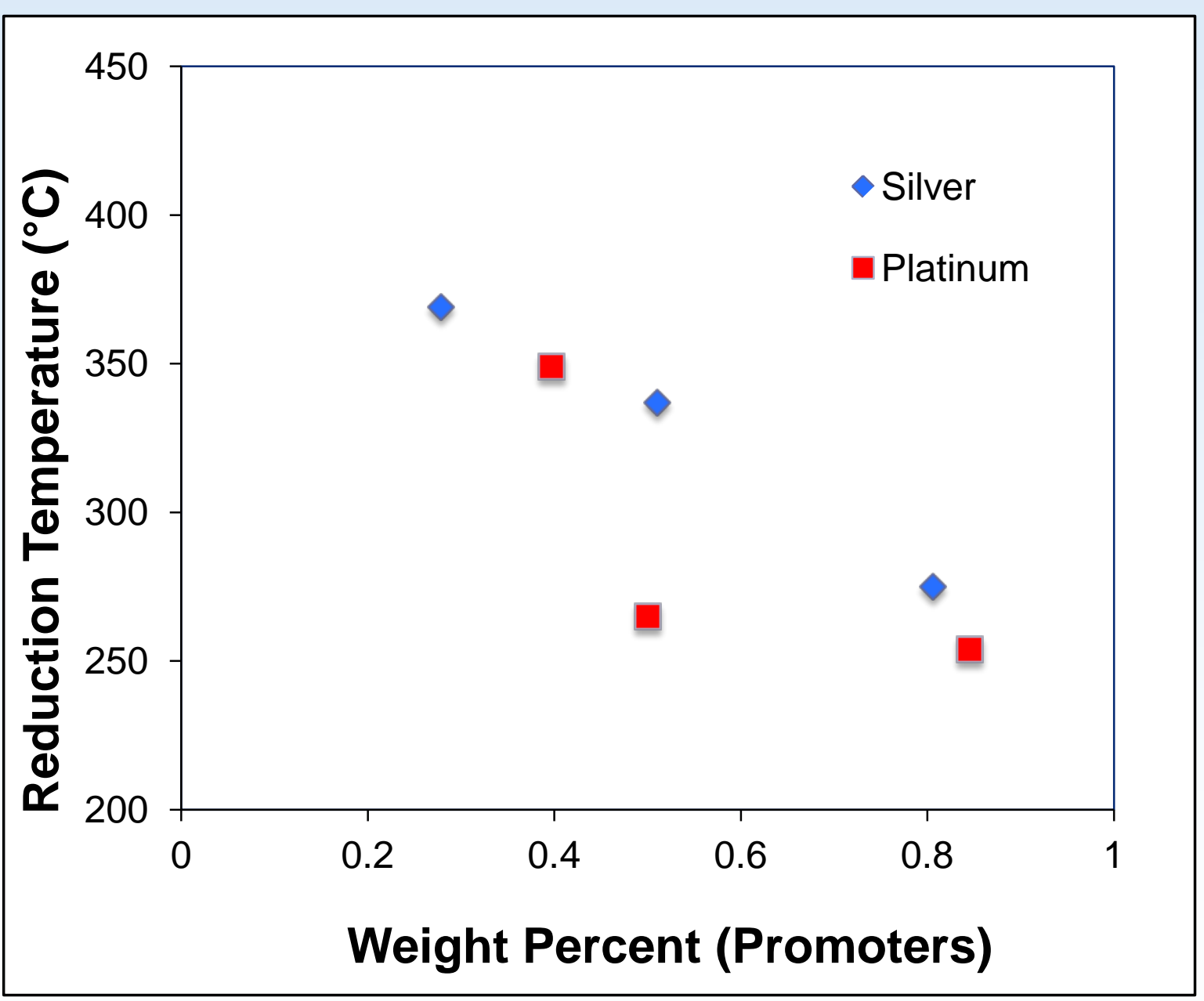




\section{SEM Images}

Surfaces look smooth and spherical - Particles look evenly dispersed!

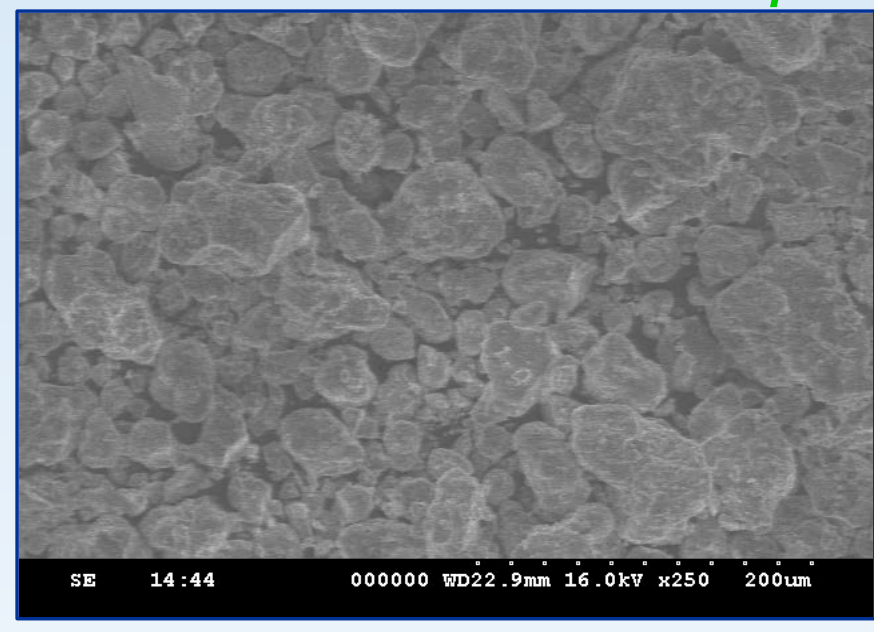

$21.6 \% \mathrm{Co}_{\mathrm{Al}} \mathrm{O}_{3}$ catalyst at $250 \mathrm{X}$

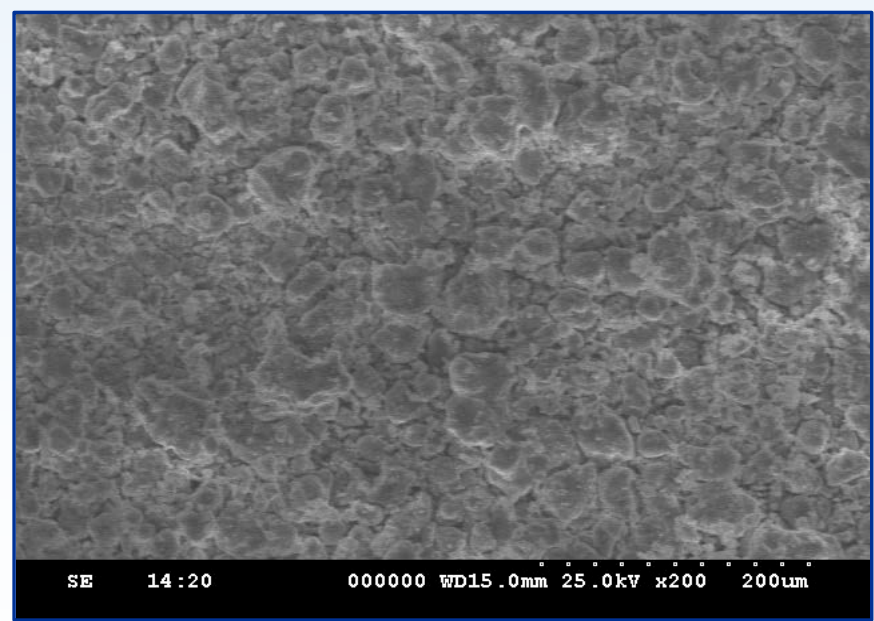

21.5\% Co/0.845\% Pt/ $\mathrm{Al}_{2} \mathrm{O}_{3}$ catalyst at $200 \mathrm{X}$

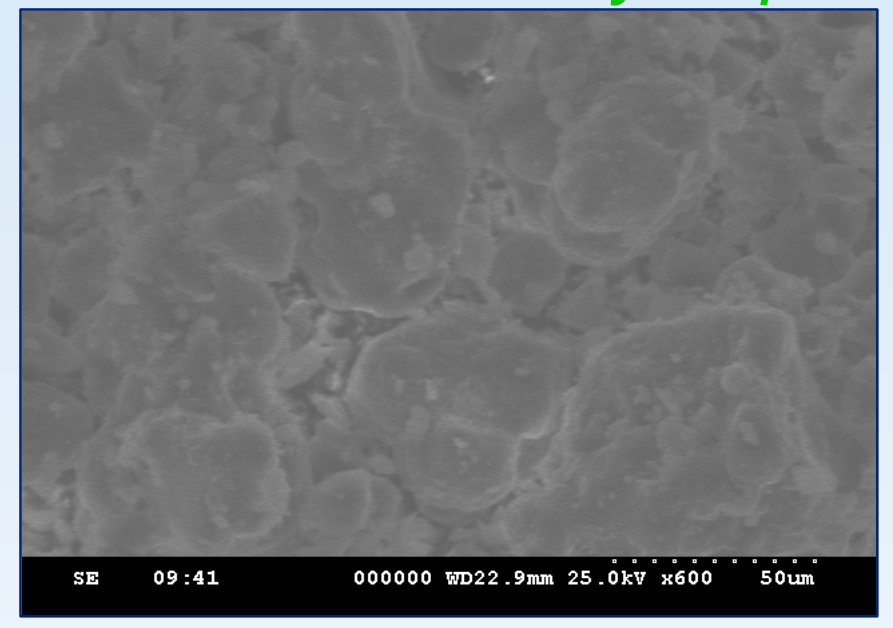

$9.31 \% \mathrm{Co} \mathrm{Al}_{2} \mathrm{O}_{3}$ catalyst at $600 \mathrm{X}$

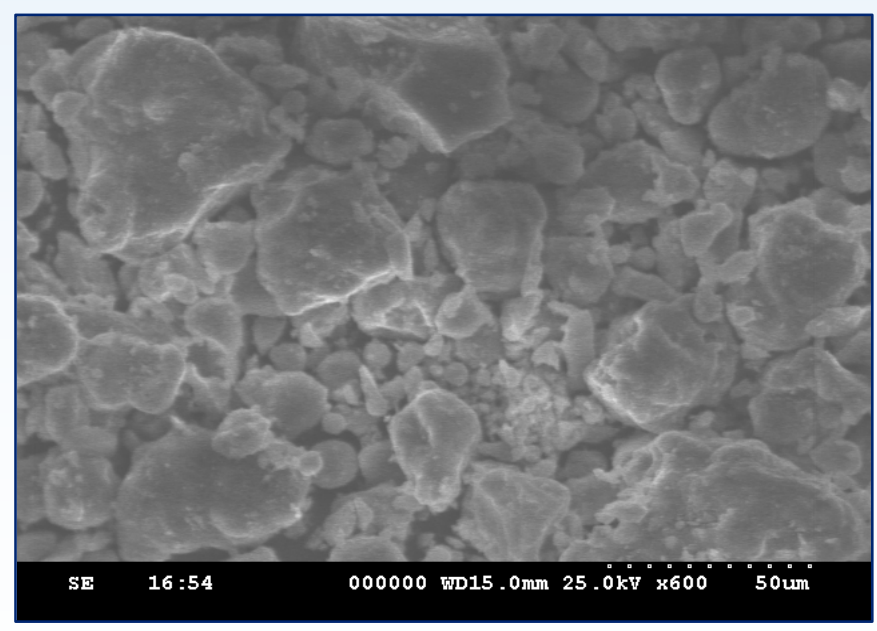

21.0\% Co/0.806\% Ag/ $\mathrm{Al}_{2} \mathrm{O}_{3}$ catalyst at $600 \mathrm{X}$ 


\section{Conclusions and Future Work}

$\checkmark$ Increase in Co loaded $\longrightarrow$ surface area decreases

$\checkmark$ Smoother Surface

$\checkmark$ Promoter attaches to surface - no increase/decrease in SA

$\checkmark$ Promoting $\mathrm{Co} / \mathrm{Al}_{2} \mathrm{O}_{3}$ decreases reduction temp.

$\checkmark$ Platinum-group metals great choice (reduces $\uparrow$ extent!)

$\checkmark$ Silver may be a good economical option

Future Work:

$>$ Investigate other promoters in platinum and coinage metals

$>$ Additional supports $\left(\mathrm{TiO}_{2}, \mathrm{SiO}_{2}\right)$

$>$ Pulse re-oxidation to investigate extent of reduction

$>$ X-Ray Diffraction (XRD) to examine crystal structure 


\section{Acknowledgements}

- Subsonic Fixed Wing program of Fundamental Aeronautics

o In-situ Resource Utilization Program of the Exploration Technology

Development and Demonstration Program

o Sasol North America

o Robyn Bradford (Central State University, NASA Academy Summer Student)

o Dr. Conrad Jones, Southern University 


\section{Questions?}

